\title{
Finite element approximation on quadrilateral meshes
}

\author{
Douglas N. Arnold ${ }^{1, \dagger}$, Daniele Boffi, ${ }^{*, \ddagger}$, Richard S. Falk ${ }^{3, \S}$ and Lucia Gastaldi ${ }^{4, \uparrow}$ \\ ${ }^{1}$ Department of Mathematics, Penn State University, University Park, PA 16802, U.S.A. \\ ${ }^{2}$ Dipartimento di Matematica, Università di Pavia, 27100 Pavia, Italy \\ ${ }^{3}$ Department of Mathematics, Rutgers University, Piscataway, NJ 08854, U.S.A. \\ ${ }^{4}$ Dipartmento di Matematica, Università di Brescia, 25133 Brescia, Italy
}

\begin{abstract}
SUMMARY
Quadrilateral finite elements are generally constructed by starting from a given finite dimensional space of polynomials $\hat{V}$ on the unit reference square $\hat{K}$. The elements of $\hat{V}$ are then transformed by using the bilinear isomorphisms $F_{K}$ which map $\hat{K}$ to each convex quadrilateral element $K$. It has been recently proven that a necessary and sufficient condition for approximation of order $r+1$ in $L^{2}$ and $r$ in $H^{1}$ is that $\hat{V}$ contains the space $Q_{r}$ of all polynomial functions of degree $r$ separately in each variable. In this paper several numerical experiments are presented which confirm the theory. The tests are taken from various examples of applications: the Laplace operator, the Stokes problem and an eigenvalue problem arising in fluid-structure interaction modelling. Copyright (c) 2001 John Wiley \& Sons, Ltd.
\end{abstract}

KEY WORDS: quadrilateral finite elements; Laplace operator; Stokes problem; fluid-structure interaction; approximation; serendipity; mixed finite element

\section{APPROXIMATION BY QUADRILATERAL FINITE ELEMENTS}

We report in this section on some results presented in Reference [1]. Let $\hat{K}$ be the unit square ]0, $1[\times] 0,1\left[\right.$; we denote by $F_{K}$ a bilinear isomorphism mapping $\hat{K}$ onto a generic quadrilateral $K$. Given a reference space $\hat{V}$ (which typically consists of polynomials), we define the finite element space on $K$ as

$$
V_{F}(K)=\left\{u: K \rightarrow \mathbb{R} \mid \hat{u}_{F} \in \hat{V}\right\}
$$

where $\hat{u}_{F}(\hat{x})=u\left(F_{K}(\hat{x})\right)$. We observe that in the general quadrilateral case if $\hat{V}$ consists of polynomials, then the functions of $V_{F}(K)$ need not be polynomials unless $F_{K}$ is affine, in which case $K$ is a parallelogram.

\footnotetext{
${ }^{*}$ Correspondence to: D. Boffi, Dipartimento di Matematica, Università Degli Studi di Pavia, Via Ferrata, 1-27100

Pavia, PV, Italy

†E-mail: dna@psu.edu

‡E-mail: boffi@dimat.unipv.it

$\S$ E-mail: falk@math.rutgers.edu

ฯE-mail: gastaldi@bsing.ing.unibs.it

Copyright (c) 2001 John Wiley \& Sons, Ltd.

Received January 2001

Accepted March 2001
} 

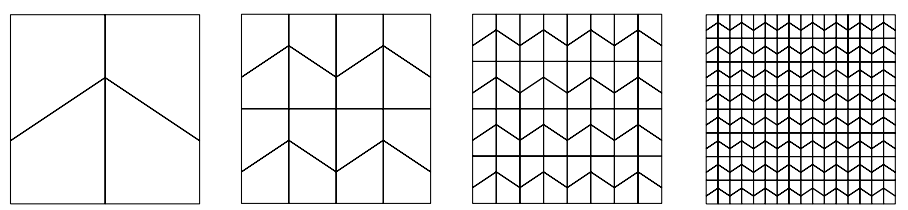

Figure 1. Meshes of self-similar trapezoids.

Given a quadrilateral mesh $\mathscr{T}$ of a two-dimensional domain $\Omega$, we denote by $V^{\mathscr{T}}$ the space consisting of functions on the domain which when restricted to an element $K \in \mathscr{T}$ belong to $V_{F}(K)$. The following theorem holds true.

Theorem 1. On a general sequence of quadrilateral meshes $\mathscr{T}_{h}$, a necessary and sufficient condition for the validity of the following estimates:

$$
\begin{array}{r}
\inf _{v \in V^{\widetilde{T}_{h}}}\|u-v\|_{L^{2}(\Omega)} \leqslant C h^{r+1}|u|_{r+1} \quad \text { for all } u \in H^{r+1}(\Omega) \\
\inf _{v \in V^{\widetilde{T}_{h}}}\left\|\nabla_{h}(u-v)\right\|_{L^{2}(\Omega)} \leqslant C h^{r}|u|_{r+1} \quad \text { for all } u \in H^{r+1}(\Omega)
\end{array}
$$

is that $\hat{V} \supseteq \mathscr{Q}_{r}(\hat{K})$, where $\mathscr{Q}_{r}(\hat{K})$ is the space of polynomials of degree less than or equal to $r$ in each variable, separately and $\nabla_{h}$ denotes the element-by-element gradient.

The sufficient part of the theorem has been proven, for instance, in References [2, 3]. The necessary part is more recent. In Reference [1] a special sequence of meshes of the unit square has been introduced. We partition the unit square $\hat{K}$ into four trapezoids. Different meshes can then be composed of translated dilates of this partition as shown in Figure 1. An important characteristic of these meshes is that all their elements are similar to each other.

In Reference [1] it has been proved that if $\mathscr{Q}_{r}(\hat{K}) \nsubseteq \hat{V}$, then neither of the estimates

$$
\inf _{v \in V(\mathscr{T} h)}\|u-v\|_{L^{2}(\Omega)}=o\left(h^{r}\right)
$$

nor

$$
\inf _{v \in V\left(\mathscr{T}_{h}\right)}\|\nabla(u-v)\|_{L^{2}(\Omega)}=o\left(h^{r-1}\right)
$$

holds, even for $u$ polynomial.

Theorem 1 shows in particular that several commonly used finite element spaces provide suboptimal approximation properties on general quadrilateral meshes. Among these, we quote in particular the serendipity (or trunk) space families $\mathscr{Q}_{r}^{\prime}$, or the mapped (or local) polynomial spaces $\mathscr{P}_{r}$. We shall comment on these spaces later on in the following sections. 

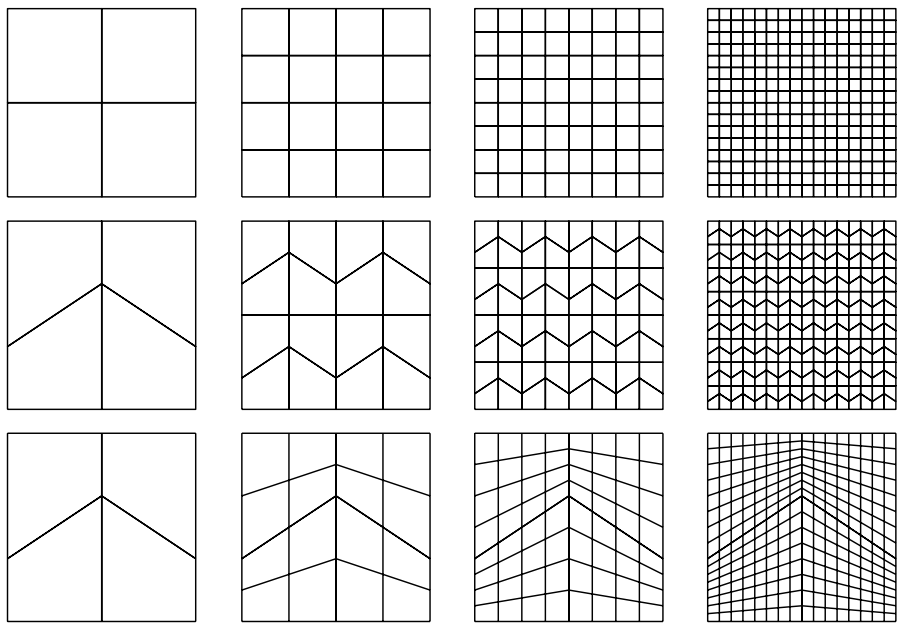

Figure 2. Three different sequences of meshes.

\section{NUMERICAL RESULTS, PART 1: THE LAPLACE OPERATOR}

In this section we report some of the numerical results presented in Reference [1]. We consider the following Dirichlet problem for the Laplace operator in the unit square $\Omega=] 0,1[\times] 0,1[$

$$
\begin{aligned}
& -\Delta u=f \quad \text { in } \Omega \\
& u=g \quad \text { on } \partial \Omega
\end{aligned}
$$

The data $f$ and $g$ are chosen such that the exact solution is the polynomial $u(x, y)=x^{3}+$ $5 y^{2}-10 y^{3}+y^{4}$. We consider three sequences of meshes: a uniform mesh of squares; the mesh of self-similar trapezoids introduced in the previous section; an asymptotically affine mesh, i.e., a sequence of meshes where the elements tend to become parallelograms (see Figure 2) as $h$ goes to zero. For each sequence of meshes we approximate problem (4) by biquadratic elements $\mathscr{Q}_{2}(\Omega)$ first and then by quadratic serendipity elements $\mathscr{2}_{2}^{\prime}(\Omega)$. We recall that the space $\mathscr{2}_{2}^{\prime}(\Omega)$ has eight degrees of freedom and is obtained from $\mathscr{Q}_{2}(\Omega)$ by dropping the term $\hat{x}^{2} \hat{y}^{2}$.

Figure 3(a) reports on the $L^{2}(\Omega)$-error for the $\mathscr{2}_{2}$ approximation: the $x$-axis and the $y$-axis represent the logarithm of $1 / h$ and of the error, respectively. The results confirm the sufficient part of Theorem 1: a clear third-order of convergence is observed. In this case we did not plot the error for the asymptotically affine mesh, since no significant difference can be observed with respect to the other two cases. On the other hand, the graph of the error for the serendipity space, plotted in Figure 3(b), clearly shows a suboptimal convergence in the case of the mesh of trapezoids: the slope of the curve is close to 3 for $h$ large, but as $h$ decreases the rate of convergence seems to tend to 2 . The results for the asymptotically affine sequence of meshes are in perfect agreement with the theory presented in Reference [1]: no loss of accuracy is present in this case. 

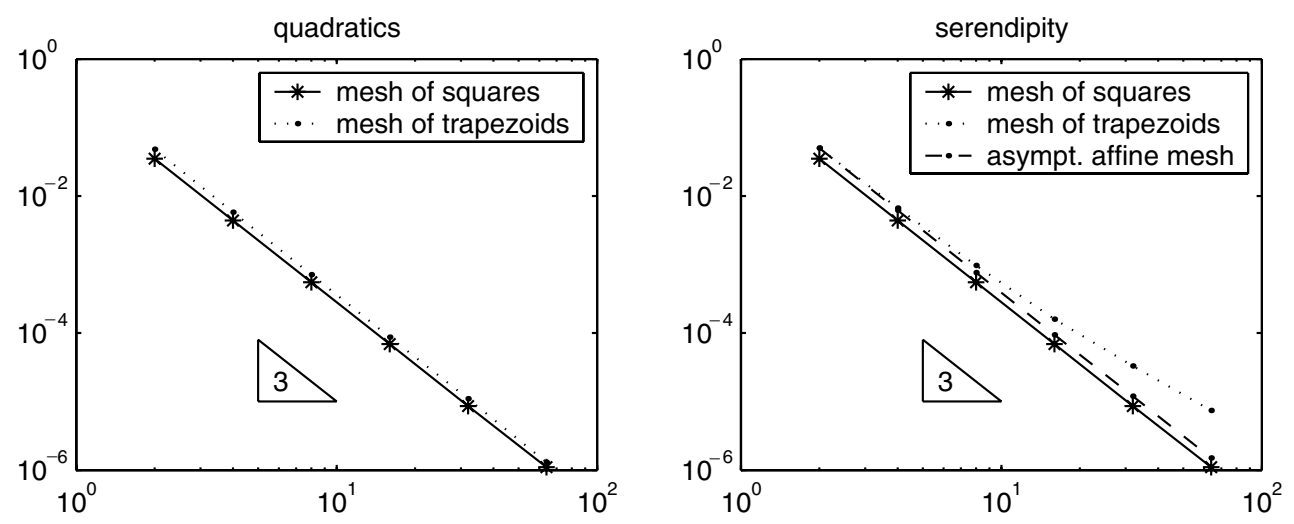

Figure 3. $L^{2}$ convergence rates: (a) for the space $\mathscr{2}_{2}$; (b) for the serendipity space.

\section{NUMERICAL RESULTS, PART 2: THE $\mathscr{2}_{2}-\mathscr{P}_{1}$ STOKES ELEMENT}

In this section we consider the approximation of the solution $(\underline{u}, p)$ to the Stokes problem

$$
\begin{aligned}
-\Delta \underline{u}+\operatorname{grad} p=\underline{f} & \text { in } \Omega \\
\operatorname{div} \underline{u}=0 & \text { in } \Omega \\
\underline{u}=0 & \text { on } \partial \Omega
\end{aligned}
$$

where $\Omega$ is the unit square $] 0,1[\times] 0,1[$. The source term $f$ is chosen such that the exact solution is given by $\underline{u}(x, y)=\left(-2 x^{2} y(1-x)^{2}\left(1-3 y+2 y^{2}\right), 2 x y^{2}(1-y)^{2}\left(1-3 x+2 x^{2}\right)\right)$ and $p(x, y)=\cos (\pi x) \cos (\pi y)$.

We use the $\mathscr{2}_{2}-\mathscr{P}_{1}$ scheme, one of the most popular Stokes elements. This method presents two possible versions (see, for instance, References $[4,5]$ ). The first choice consists in a local (or 'mapped')-pressure approximation, i.e., the discrete pressures are defined on the reference square as linear functions and mapped onto the generic quadrilateral as described in (1). This choice, according to the theory presented in the previous section, cannot provide optimal convergence order on general quadrilateral meshes, since the reference space does not contain all of $\mathscr{Z}_{1}$. The second possibility is to define the discrete pressures $p$ such that the restriction $\left.p\right|_{K}$ is a linear function for any quadrilateral $K$ of the considered mesh. For this definition we did not make use of the bilinear mappings $F_{K}$. This second choice is known as global (or 'unmapped')-pressure approximation. The terminology global/local refers to the choice of the co-ordinate system: with the local approach one can define the discrete pressures via a local co-ordinate system, while with the global one the pressures are built as piecewise linear polynomials with respect to the global co-ordinate system.

According to Reference [6], the $\mathscr{Q}_{2}-\mathscr{P}_{1}$ element has been introduced during a conference in 1979. The analysis for the global approach can be found, for instance, in References [7-9] and for the local approach in Reference [5].

For our computations we shall make use of the same sequences of meshes as in the previous section (see Figure 2). The results for the pressures (plot of the $L_{2}$ error) are presented 

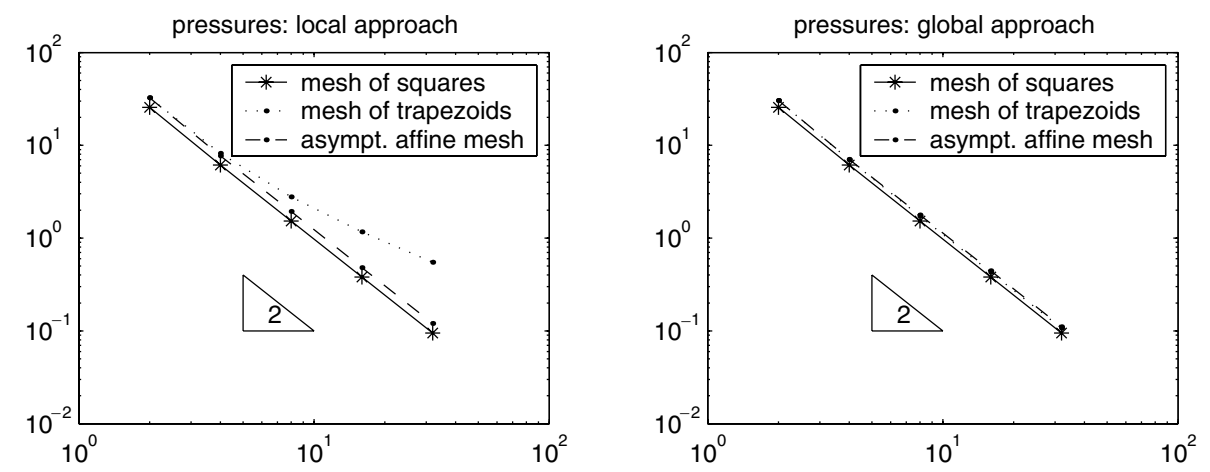

Figure 4. $L^{2}$ pressure error estimate: (a) local approach; (b) global approach.
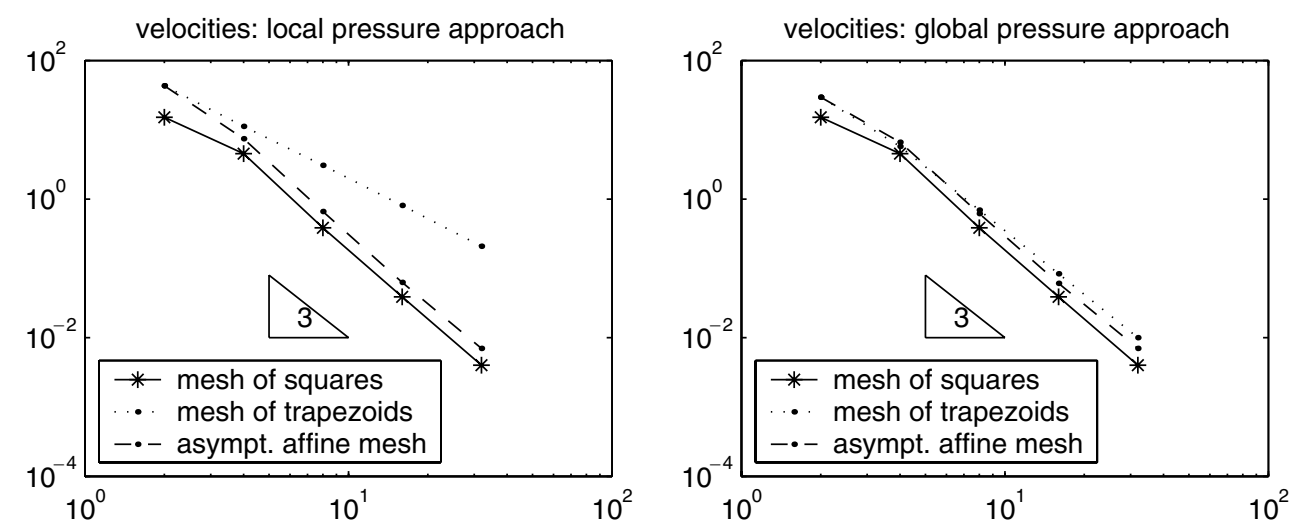

Figure 5. $L^{2}$ velocity error estimate: (a) local pressure approach; (b) global pressure approach.

in Figure 4. As expected, the global approach provides optimal second-order convergence for all possible meshes, while the local-pressure approximation is only first-order accurate on the distorted trapezoids mesh. Both methods are second-order convergent (indeed they are equivalent) with the mesh of squares and second-order convergence also holds for the asymptotically affine mesh.

Figure 5 reports the $L^{2}$ errors of the velocities. We remark that both methods use the same approximating space for the velocity, namely the space of continuous piecewise biquadratic functions $\mathscr{Q}_{2}$ for each component. In particular, this space should provide the optimal thirdorder of convergence in $L^{2}$. However, the results turn out to be suboptimal in the case of the local-pressure approximation on the distorted mesh. This is due to the fact that the error estimates of mixed methods link together the approximation properties of velocities and pressures. Hence, in the local approach, a bad approximation of the pressures produces a negative effect for the approximation of the velocities too. 


\section{NUMERICAL RESULTS, PART 3: AN EIGENVALUE PROBLEM}

In this last section we report the results of our computation for the approximation of the eigenmodes $(\underline{u}, \lambda)$ of the following problem:

$$
\begin{array}{rlrl}
-\operatorname{grad} \operatorname{div} \underline{u}+s \operatorname{curl} \operatorname{rot} \underline{u} & =\lambda \underline{u} & & \text { in } \Omega \\
\underline{u} \cdot \underline{n}=0 & & \text { on } \partial \Omega
\end{array}
$$

Here $\Omega$ is still the unit square $] 0,1[\times] 0,1[, \partial \Omega$ its boundary, and $\underline{n}$ denotes the outward oriented normal unit vector. The positive number $s$ is a penalization parameter. For a discussion on how to choose this parameter we refer, for instance, to Reference [10]. In our numerical tests we will take $s=10$. This problem has been intensively studied in the literature [11-14]. It arises, for instance, as a part of a fluid-structure interaction problem. Interchanging the role of the curl with the grad and of the rot with the div it can also been viewed as a penalty method for the computation of the Maxwell's cavity eigenvalues [10].

To our best knowledge, at the present time no efficient numerical method has been proposed yet for the approximation of problem (5). Among the known schemes we test the $\mathscr{Q}_{2}-\mathscr{P}_{1}-\mathscr{P}_{1}$ three-field method proposed by Bathe et al. in Reference [11]. For a presentation of the method and a partial analysis of it, we refer to the original paper [11] and to Reference [10]. In this scheme, the eigenfunctions $\underline{u}$ are approximated by means of continuous piecewise biquadratic vector fields and two auxiliary variables (representing $\operatorname{div} \underline{u}$ and $\operatorname{rot} \underline{u}$ ) are approximated by piecewise linear functions.

As in the previous section, we have two different choices for the definition of the linear spaces: either a local (or 'mapped') approach or a global (or 'unmapped') approximation. In Figures 6 and 7 we show the convergence history of the first two eigenvalues using the two schemes and two different sequence of meshes: the mesh of squares and the mesh of selfsimilar trapezoids (see Figure 2). While the first eigenvalue is well approximated in all the considered cases, this is not the case for the second one. Using the local approach there is no
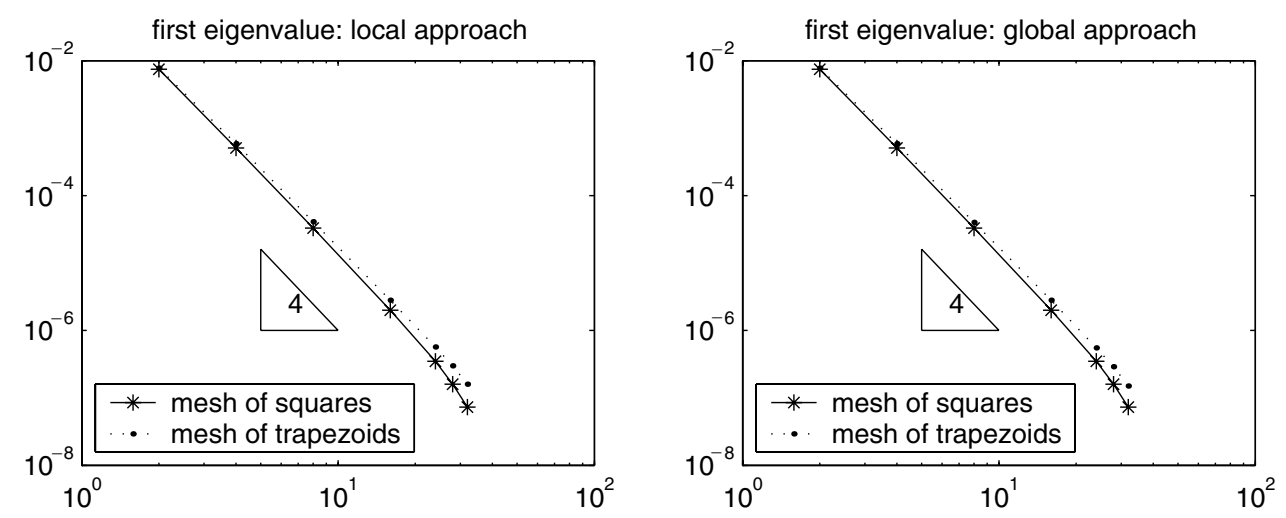

Figure 6. First eigenvalue computed using: (a) the local approach; (b) the global approach. 

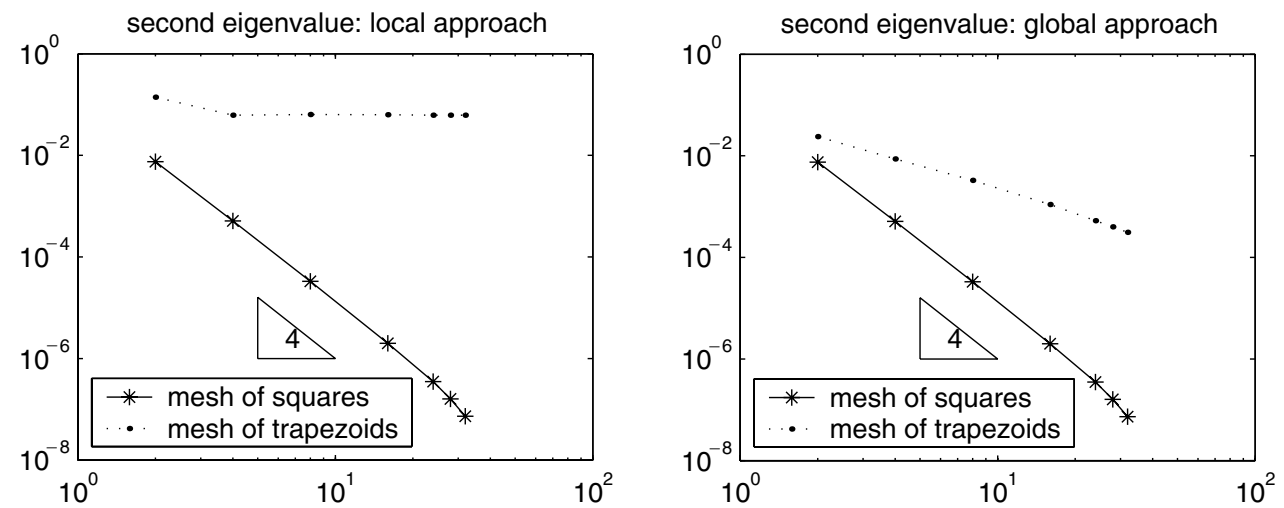

Figure 7. Second eigenvalue computed using: (a) the local approach; (b) the global approach.

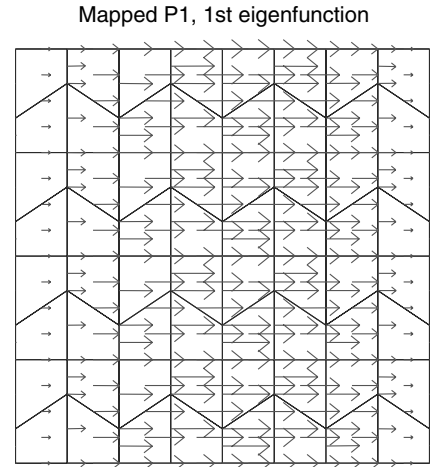

Unmapped P1, 1st eigenfunction

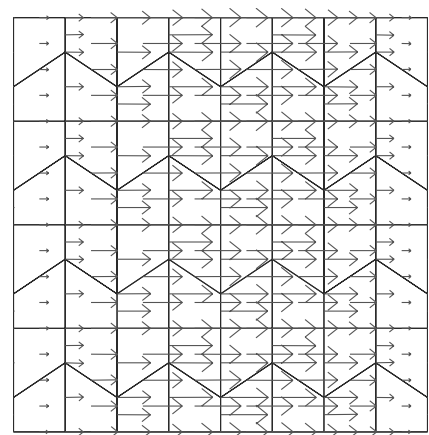

Mapped P1, 2nd eigenfunction

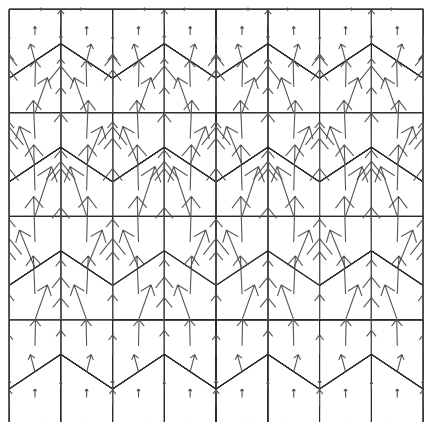

Unmapped P1, 2nd eigenfunction

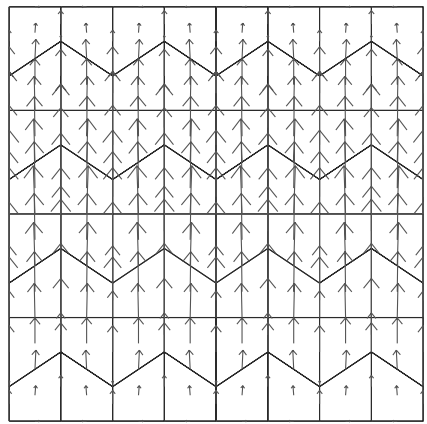

Figure 8. The computed eigenfunctions.

convergence on the mesh of trapezoids (the solution seems to be stable but not convergent) and using the mesh of squares the convergence is only suboptimal.

The interpretation of these results is unclear. The difference between the local and the global approach seems to be due to the different approximation properties, as shown for the 
Stokes problem in the previous section. On the other hand, even in the better case the results are not satisfactory and this phenomenon needs to be further investigated.

We conclude this section by showing in Figure 8 the corresponding computed eigenfunctions. It is apparent that the anisotropy of the mesh makes the difference between the approximation of the first and the second eigenmode.

\section{CONCLUSIONS}

In this paper, we presented some numerical experiments related to the theory illustrated in Reference [1]. The first example concerns the approximation of the Laplace operator with biquadratic or serendipity elements. The numerical experiments show that on general quadrilateral meshes the optimal convergence rate is achieved only with full biquadratics.

The second and third examples deal with the discontinuous piecewise linear element. On general quadrilaterals, a local or a global approach can be used. According to the theory of Reference [1], only the global approach can provide optimal approximation properties if the mesh is far from being affine. In one case $\left(\mathscr{Q}_{2}-\mathscr{P}_{1}\right.$ Stokes element) the numerical results clearly confirm the theory; while in the last example $\left(\mathscr{Q}_{2}-\mathscr{P}_{1}-\mathscr{P}_{1}\right.$ element for an eigenvalue problem) the results are unsatisfactory and need further investigation.

\section{REFERENCES}

1. Arnold DN, Boffi D, Falk RS. Approximation by quadrilateral finite elements. Mathematics of Computation 2001; at press.

2. Ciarlet PG, Raviart P-A. Interpolation theory over curved elements with applications to finite element methods. Computation Methods in Applied Mechanical Engineering 1972; 1:217-249.

3. Ciarlet PG. The Finite Element Method for Elliptic Problems. North-Holland: Amsterdam, 1978.

4. Gresho P, Sani R, Engelman MS. Incompressible Flow and the Finite Element Method Advection-Diffusion and Isothermal Laminar Flow. Wiley: West Sussex, U.K. 1998.

5. Boffi D, Gastaldi L. On the quadrilateral $Q_{2}-P_{1}$ element for the Stokes problem. Pubbl. IAN-CNR 1194/00, 2000.

6. Brezzi F, Fortin M. Mixed and Hybrid Finite Element Methods. Springer: New York, 1991.

7. Stenberg R. Analysis of Mixed Finite Elements Methods for the Stokes Problem: A Unified Approach. Mathematical Computing 1984; 42(165):9-23.

8. Girault V, Raviart P-A. Finite Element Methods for Navier-Stokes Equations, Theory and Algorithms. Springer: Berlin, 1986.

9. Girault V. A local projection operator for quadrilateral finite elements. Mathematical Computing 1995; 64(212):1421-1431.

10. Boffi D, Chinosi C, Gastaldi L. Approximation of the grad div operator in non-convex domains. CMES 2000; 1:27-38.

11. Bathe K-J, Nitikitpaiboon C, Wang X. A mixed displacement-based finite element formulation for acoustic fluid-structure interaction. Computers and Structures 1995; 56:225-237.

12. Bermúdez I, Durán R, Muschietti A, Rodríguez R, Solomin J. Finite element vibration analysis of fluid-solid systems without spurious modes. SIAM Journal of Numerical Analysis 1995; 32:1280-1295.

13. Rodríguez R, Solomin JE. The order of convergence of eigenfrequencies in finite element approximations of fluid-structure interaction problems. Mathematical Computing 1996; 65(216):1463-1475.

14. Bermúdez A, Durán R, Rodríguez R. Finite element solution of incompressible fluid-structure vibration problems. International Journal for Numerical Methods in Engineering 1997; 40(8):1435-1448. 